\title{
Comparación de marcos conceptuales de la teoría de la discriminación de J. Rodríguez Zepeda y Adela Cortina.
}

Comparison of conceptual framework of Rodriguez Zepeda and Adela Cortina's theory.

Yazmin Vargas Valenzuela

Licenciatura en Filosofía. Universidad de Guadalajara (MÉXICO)

CE: yazvv8@gmail.com

Cynthia Alejandra Santana Calleros

Licenciatura en Filosofía. Universidad de Guadalajara (MÉXICO)

Eduardo Netzahualcoyotl Torres Hernandez

Licenciatura en Filosofía. Universidad de Guadalajara (MÉXICO)

\section{Sofía Gutiérrez Pérez}

Instituto de Investigación de Derechos Humanos

de la Comisión Estatal de Derechos Humanos de Jalisco (MÉXICO)

CE: gutierrez sofia@hotmail.com

DOI: $\underline{10.32870 / \text { sincronia.axxiv.n77.22a20 }}$

Esta obra está bajo una Licencia Creative Commons Atribución-NoComercial 4.0 Internacional

CC) $\mathrm{BY} \cdot \mathrm{NC}$

Recibido: 10/01/2019

Revisado: $19 / 03 / 2019$

Aprobado: 25/09/2019

\section{RESUMEN}

El presente escrito comienza con la construcción del contexto de la discriminación en México para aterrizar en la situación de éste en la entidad de Jalisco. Una vez montado el contexto, se señala la aportación que el presente ensayo puede proporcionar al problema de la discriminación del país. En 
efecto, se desarrolla la comparación del marco teórico de Zepeda con el de Adela cortina para formular un entendimiento más integral del fenómeno de la discriminación con el fin de disminuir o eliminarlo de nuestro país.

Palabras clave: Discriminación. Trato. Pobre.

\section{ABSTRACT}

The present work begins with the construction of the context of discrimination in Mexico in order to land in the situation of it in the state of Jalisco. Once the context is built, it is pointed out the contribution that the present article can add to the problem of discrimination in the country. In effect, it is compared Zepeda's theoretical framework and Cortina's with the goal of formulating a better understanding of the discrimination phenomena for decreasing or eliminating it from our country.

Keywords: Discrimination. Treatment. Poor.

La igualdad y no discriminación es un derecho inherente a todas las personas ¿Cuáles son las implicaciones de esto? Que todos los seres humanos tienen el derecho a ser iguales en dignidad, a ser tratados con respeto y consideración y a participar sobre bases iguales en cualquier área de la vida económica, social, política, cultural o civil (Naciones Unidas, 2015). Estos son principios establecidos internacionalmente en la Declaración Universal de los Derechos Humanos reconocidos por el Estado mexicano. Este reconocimiento se encuentra consolidado en que el Estado mexicano ha suscrito y ratificado decenas de instrumentos internacionales de protección de los derechos humanos del sistema internacional de la Organización de las Naciones Unidas y del sistema interamericano, integrado por la organización de los Estados Americanos (Consejo para Prevenir y Eliminar la Discriminación de la Ciudad de México, s.f.). Este reconocimiento se convierte oficial en junio de 2011 con la reforma constitucional en materia de derechos humanos, en que los tratados internacionales pasan a ser parte de nuestro derecho interno, colocando los derechos humanos como 
eje fundamental en el que debe girar la acción pública. A nivel nacional, el derecho a la igualdad y no discriminación es el artículo 1o de nuestra constitución. Su párrafo tercero indica:

Queda prohibida toda discriminación motivada por origen étnico o nacional, el género, la edad, las discapacidades, la condición social, las condiciones de salud, la religión, las opiniones, las preferencias sexuales, el estado civil o cualquier otra que atente contra la dignidad humana y tenga por objeto anular o menoscabar los derechos y libertades de las personas (Congreso de la Unión, 2011).

En efecto, todas las personas en México tienen el derecho a ser iguales en dignidad y ser tratados por igual ante la ley. Desafortunadamente, en los hechos, las personas se presentan con obstáculos para tener acceso a este derecho fundamental. Entre los principales obstáculos que lastiman a la sociedad mexicana esta la discriminación porque, como forma grave de desigualdad de trato, “ [...] niega el ejercicio igualitario de libertades, derechos y oportunidades a cualquier persona" (Consejo Nacional para Prevenir la Discriminación, 2011, p. 6), desembocando en la exclusión inmerecida e injusta de personas del estado de derecho. Esto pone a las personas discriminadas en desventaja para desarrollar de forma plena su vida, además de colocarlas en una situación de alta vulnerabilidad. Las personas que padecen esta desventaja sistemática son cada vez más susceptibles a la violación de sus derechos en el futuro (Consejo Nacional para Prevenir la Discriminación, 2011).

Hace más de quince años, "el Estado mexicano admitió que la discriminación era un problema Público" (Merino y Vilalta, 2014, p. 438). Desde entonces, se han emprendido una serie de acciones para prevenir y erradicar la discriminación en nuestro país. Los primeros esfuerzos por construir normas jurídicas capaz de proteger los grupos afectados por la discriminación se rastrean en la acción realizada por la Comisión Ciudadana de Estudios contra la Discriminación en marzo del 2001. En este año, la comisión convoca reuniones periódicas en foros plurales para exponer las causas más graves y recurrentes de discriminación. La Comisión Ciudadana tuvo dos objetivos: formular un proyecto legal amparado en la prohibición constitucional de toda forma de discriminación y la propuesta de un Consejo Nacional para Prevenir la Discriminación como órgano de Estado. Meses posteriores a estas 
reuniones, el 14 de agosto del 2001 se incluye en el marco constitucional el derecho fundamental a no ser discriminado mediante la adición del párrafo tercero al artículo $1^{\circ}$ de la Constitución Política de los Estados Unidos Mexicanos (Congreso de la Unión, 2001). En el 2003 se promulga la Ley Federal para Prevenir y Eliminar la Discriminación (COPRED, s.f.) y el año siguiente se crea el Consejo Nacional para Prevenir la discriminación (Consejo Nacional para Prevenir la Discriminación, 2011).

En el 2005, se construye La primera Encuesta Nacional sobre Discriminación en México. Ésta, como comenta Zepeda, expresidente académico de la Cátedra UNESCO "Igualdad y no discriminación", dejó en evidencia que nuestra sociedad abriga poderosos componentes de misoginia, racismo, xenofobia y homofobia. Escasas variaciones de esto arroja la segunda encuesta nacional realizada en 2010. En términos generales, nuestra cultura nacional, Zepeda concluye, se caracteriza por el rechazo a lo diferente (Zepeda, 2011).

La Enadis más reciente del 2017 muestra que nuestra cultura sigue abrigando fuertes componentes discriminatorios. Esta revela que una de cada cinco personas ha sido discriminada. Los grupos más afectado son aquellos que han sido históricamente vulnerables como los indígenas, las personas con discapacidad, las mujeres, los jóvenes y trabajadoras domésticas. Cuatro de cada 10 personas indígenas declaró que fue discriminada por su origen étnico, 58.3\% de las personas con discapacidad por su condición y $41.7 \%$ sufrió esta situación por sus creencias religiosas. Por género, $51 \%$ de las mujeres dijo haber sido discriminada por su apariencia, 32\% por su religión, 30\% por su sexo y $17 \%$ por su manera de hablar. En el caso de los hombre, la cifra fue mayor solamente por apariencia con 56\%. La brecha más significativa (arriba de 24 puntos las mujeres) entre ambos sexos se encuentra cuando se habla de discriminación por sexo. Las trabajadoras domésticas son uno de los sectores más vulnerables, nueve de cada 10 destacó que su labor es poco valorada por la gente, 87.7\% mencionó que no cuenta con prestaciones laborales y $57.1 \%$ de ellas dijo que se respetan poco o nada sus derechos (Instituto Nacional de Estadísticas y Geografía, 2018a) .

Por motivo de apariencia, el $53.8 \%$ de la población de 18 años y más ha percibido discriminación en el último año anterior de la encuesta (p.7). El tono de piel es uno de los principales motivos de discriminación en el país. La encuesta muestra que entre más oscuro sea el tono de piel, 
disminuye el nivel de escolaridad. El $30.4 \%$ de las personas con tonos de piel más claros tienen estudios de nivel superior; $22.7 \%$ de las personas con tono intermedio cursaron este nivel educativo y $16.2 \%$ de quienes tienen piel oscura (Instituto Nacional de Estadísticas y Geografía, 2018b).

La encuesta también muestra los porcentajes de discriminación por entidad federativa. El estado de Jalisco casi se posiciona (por solo 2.7 puntos) entre las entidades con mayor porcentaje de discriminación. 21.3\% de la población jalisciense de 18 años o más declaró haber sido discriminada por algún motivo o condición. Se consideraron estados con mayor prevalencia de discriminación a partir del 24\% (Instituto Nacional de Estadísticas y Geografía, 2018b). Otro foco alarmante está en el hecho de que Jalisco "se mantiene en el tercer lugar nacional en quejas por discriminación" (El Informador, 2018). Además, en la entidad se siguen presentando casos explícitos de discriminación. En el cruce de Mariano Otero y Guadalupe, niños indígenas fueron puestos a lavar baños tras pedir acceso en una primaria en este año en curso (Gutiérrez, 2018).

Es importante mencionar las acciones recientes de la entidad para prevenir y eliminar la discriminación. En diciembre del 2015, entra en vigor la Ley Estatal para Promover la Igualdad, Prevenir y Eliminar la Discriminación en el Estado de Jalisco (Congreso del Estado de Jalisco, 2016). Posterior a la publicación de la ley en el Periódico Oficial de Jalisco, se crea el Consejo para Prevenir y Eliminar la Discriminación. En octubre del 2017, Guadalajara firma convenio con Conapred para crear un programa municipal para la igualdad y la No Discriminación (Arenas, 2017).

Con toda la lucha y los esfuerzos iniciados hace más de quince años para enfrentar la discriminación, la ENADIS 2017 muestra que todavía queda mucho por hacer en el tratamiento de este problema público tanto a nivel nacional como estatal.

¿Qué es lo que hay que hacer? ¿Cuales son las acciones que el gobierno ha de llevar acabo? Hay un libro publicado por el Consejo para Prevenir y Eliminar la Discriminación de la Ciudad de México (2013) que lleva por título Antología de la Política Pública Antidiscriminatoria 2013-2017. Este tiene como objetivo principal averiguar los logros en el ámbito de las políticas públicas, los retos que ha presentado para la Administración Pública y las áreas de oportunidad en las que se tendrá que continuar trabajando para disminuir el distanciamiento entre los hechos y el derecho a la igualdad y 
no discriminación. Sin embargo, este libro contiene información relevante para abonar a la solución de las preguntas antes expuestas. La Antología proporciona esclarecimientos sobre la manera de configurar una adecuada intervención del gobierno para enfrentar el problema público de la discriminación y desigualdad. Se expone una explicación de lo que es un problema público y señala la estrecha relación que se guarda entre éste y la planeación de los cursos de acción para solucionarlo. Se indica que puede tenderse a pensar que un problema público consiste en articular en palabras el problema y a caracterizarlo a partir de sus efectos cuantitativos o cualitativos sobre la vida de las personas. Sin embargo, el reconocimiento de un problema público no se limita al simple señalamiento de la problemática. Este busca identificar las causas que generan tales problemáticas y señalar sus consecuencias. A la par, se busca segmentar analíticamente dichas causas y consecuencias e intentar encontrar efectos causales que permitan trazar posibles cursos de acción. El Estado, en el cumplimiento de su obligación de garantizar el derecho a la igualdad y no discriminación, habrá de seleccionar tales cursos de acción para delinear su intervención pública tomando en consideración los recursos y capacidades con las que se cuentan para asegurar que sean intervenciones factibles. En efecto, "la definición del problema debe decirnos de qué se trata la intervención del Estado, a dónde quiere llegar y qué efectos está buscando; debe servirnos para reconocer la relación entre las causas identificadas y las soluciones propuestas" (citado en la Antología de política pública antidiscriminatoria 2013-2017, s.f., p.20).

La explicación del problema público demuestra que es indispensable definir las causas, consecuencias y el problema en sí mismo para trazar líneas de acción. De esta manera, se puede categorizar un problema público en dos momentos: 1) esclarecimiento de la naturaleza, causa y consecuencias del problema público y 2) trazar las líneas de acción para solucionarlo.

Una acción del gobierno para intervenir públicamente es mediante la construcción de políticas públicas. Si se parte de la definición del problema público antes expuesto, se deduce que la política pública tiene tres características. Merino las expone de la siguiente manera:

1) en [las políticas públicas] se definen, de forma previa y precisa, un problema público, 2) presentan argumentos respecto a la manera en cómo intervendrá la autoridad pública para 
resolverlo e 3) identifican claramente los resultados esperados en un tiempo determinado (Citado en la Antología de política pública antidiscriminatoria 2013-2017, s.f, p.19).

Expuesto lo anterior, se infiere que el trazo adecuado de los cursos de acción de la política pública dependen innegablemente de una correcta descripción del problema como de sus causas y consecuencias. Por ejemplo, si las causas, consecuencias y el problema de la discriminación en sí mismo están correctamente definidos, es decir, si el discurso teórico corresponde a la realidad, es razonable creer que la configuración de las líneas de acción serán las idóneas; de lo contrario, los cursos de acción no resolverán el problema. Esto puede quedar mejor entendido con el siguiente ejemplo similar. Piénsese en el caso de un doctor que diagnostica erróneamente a su paciente. En vez de detectarle pulmonía, le diagnostica tos severa. Así, le receta medicamento para dicha tos en ves para la pulmonía ¿Cuál será el resultado de esto? Que el paciente no se cure. Este ejemplo sirve como muestra de la gran importancia del correcto entendimiento del problema. Es difícil el creer que unas líneas de acción desconocedoras del problema puedan solucionarlo.

El entendimiento de un problema se realiza mediante un marco teórico ${ }^{1}$. Éste tiene la función equivalente a las de una gafas por medio de las cuales vemos la realidad. El marco teórico interpreta los datos observados, es decir, el problema adquiere su forma (la descripción de sus causas, consecuencia y naturaleza misma) mediante éste.

El presente ensayo reconoce la importancia de partir de un adecuado marco teórico mediante el cual se proporcione un entendimiento acertado de las causas, consecuencias como del problema de la discriminación en sí mismo. Es importante el aclarar que nunca se podrá encontrar un marco teórico acabado. Éste está en constante transformación como la realidad misma lo está. En base a dicho reconocimiento y mediante herramientas analíticas se aportará en el tratamiento del problema de la discriminación reflexiones que abonen en la construcción de un óptimo marco teórico del fenómeno de la discriminación. Esta tarea requiere del trabajo conceptual y analítico de los estudios

\footnotetext{
1 “Provee un marco de referencia para interpretar los resultados de estudio" (Marco Teórico, s.f.)
} 
antidiscriminatorios. Con tal fin, se realizará un contraste entre dos marcos teóricos: uno localizado en el libro La otra desigualdad: La discriminación en México, escrito por el filósofo Jesús Rodríguez Zepeda quien fungió como presidente académico de la Cátedra UNESCO "Igualdad y no discriminación" (2010-2012) auspiciada por la Universidad de Guadalajara y el Consejo Nacional para Prevenir la Discriminación. El otro marco teórico es el expuesto en el libro Aporofobia, el rechazo al pobre; un desafío para la democracia de Adela Cortina Orts quien es una filósofa española ganadora del Premio Internacional de Ensayo Jovellanos 2007, catedrática de Ética de la Universidad de Valencia entre otras funciones.

\section{La otra desigualdad: La discriminación en México}

Antes de entrar de lleno a la descripción del fenómeno de la discriminación, se aclara que la intención de construirles los siguientes marcos conceptuales va acompañada del deseo de mostrárselos de la manera más simple posible para su fácil comprensión más allá del ámbito de académicos especializados.

¿Qué es la discriminación? Es un tipo de desigualdad: desigualdad de trato. El trato es un elemento clave para entender la compleja fenomenología de la discriminación. Dediquemos unos minutos a llevar la atención a ese elemento que refiere a la manera en que se dirige un ser a otro en la interacción. Por ejemplo, cuando se está frente a un cachorro, no se le invita a tomar asiento para desayunar en la mesa ¿Por qué? Porque se sabe de antemano que los cachorros no tienen la capacidad de sentarse en una silla, es decir, esta idea que poseemos previo a la interacción orienta la manera en que nos dirigiremos al cachorro. En el caso de la discriminación, las ideas que desembocan en el trato diferenciado se llaman estigmas y prejuicios. Estos preexisten al acto de interactuar, "pero preexisten ¿dónde?" -puede que surja la cuestión. Indudablemente, estos estigmas y prejuicios residen en la mente; más en específico, en el terreno de las representaciones colectivas del orden social que filósofos han llamado cosmovisión y sociólogos y antropólogos le han dado por nombre imaginario colectivo. Lo que es importante tener en claro del imaginario social o cosmovisión es que 
estas representaciones son compartidas por un colectivo y establecen el orden que la sociedad ha de tener, es decir, son intersubjetivas y de carácter normativo.

¿Qué, en específico, es referido por "representaciones? Alguien que es ajeno al lenguaje utilizado en filosofía le puede venir de inmediato en mente el nombre de un director o representante de una compañía o simplemente sospechar que la palabra está ligada a algo que es representativo en un grupo de personas. La palabra no está tan distanciada de aquello, pero en este caso, se utiliza en el ámbito de las ideas, es decir, refiere a la idea representativa de algo. Hay un filósofo que se llama Husserl quien realiza reflexiones profundas entorno a las representaciones. Morente (2013) explica alguna de ellas a la vez que expone la definición de representaciones de Husserl en su libro Lecciones preliminares de filosofía. Él nos dice que para Husserl, las representaciones deben ser miradas desde dos puntos de vista: el psicológico y como referencia intencional a un objeto. Si se le mira a la representación desde el punto de vista psicológico, se notará que tiene una individualidad psicológica -maneras propias del sujeto de percibir, pero si se le concibe desde el segundo punto de vista, se nota que esa representación singular es el representante de un objeto -por más variadas que sean las maneras de representarse el objeto, no se deja de representar tal objeto. Morente explica esto con el ejemplo del objeto Napoleon:

Así, si yo quiero pensar el objeto Napoleon, no puedo pensarlo más que representándome a Napoleon; pero la representación que yo tengo de Napoleón tendrá que ser singular: o bien me lo represento montado a caballo en el puente de Arcole, o bien me lo represento en la batalla de Austerlitz con la cabeza baja y la mano puesta en su redingote; o bien me lo represento desesperado, después de la derrota de Waterloo. Cada una de estas representaciones por sí misma es singular, pero las tres, aunque sean totalmente distintas unas de las otras, se refieren al mismo objeto, que es Napoleon (Morente, 2013, p.46).

En el caso de las representaciones colectivas del orden social que fungen como motor de actos discriminatorios, son las definiciones colectivamente compartidas de la naturaleza de grupos donde yace la raíz de los actos discriminatorios. Por ejemplo, en el caso de México, la encuesta ENADIS 2017 
muestra que $60.3 \%$ de los encuestados opinan que los jóvenes son irresponsables (Baylón, 2018). Es decir, el 60.3\% de los encuestados se representan al grupo social de los jóvenes como irresponsables. El pensamiento que se les viene a la mente de inmediato al pensar en un joven es de irresponsabilidad. Ésta es una característica que se adjudica indiscriminadamente a un joven por su condición de ser joven. No se le observa en su vida cotidiana y no se identifican las razones por las que faltan a sus responsabilidades con el fin de ponerlas en tela de juicio, para poder formular el juicio de que es irresponsable. Sino que simplemente cuando se le mira que es joven se da por hecho de que es irresponsable, como si ser joven fuera sinónimo de irresponsabilidad.

Entonces, vemos que el trato no surge por mera espontaneidad. Este es resultado de ideas preconcebidas y compartidas. En el caso de la discriminación, el trato desigual es el resultado de estigmas y estereotipos que residen en el terreno de las representaciones colectivas del orden social.

¿Cómo es que se forman estos estigmas y prejuicios? Estos son productos de la cultura, pues los esquemas culturales son los que forman a sujetos morales y sociales, y a su vez los esquemas de conducta. Es en este punto que Zepeda introduce el término instituciones para referir a las prácticas sociales (esquemas de conducta) regularizadas o estandarizadas por "clase de actores", "es decir, grupos o tipos de personas, definidos culturalmente por elementos lingüísticos y simbólicos compartidos" (Zepeda, 2011, p.24). Entre las instituciones informales se encuentran los valores culturales, familiares o de moralidad social, mientras que en las instituciones formales se expresan como norma legal o como principio de institucional formal. Por tanto, la discriminación es un problema que proviene de la cultura con naturaleza estructural debido a las prácticas relativamente estables que la sostienen.

En base a esta exposición del fenómeno de la discriminación, Zepeda propone para disminuir o eliminarla, la construcción política de estrategias de condición también institucional y estructural que implica la creación de instituciones formales en la sociedad como organismos, leyes, normas, etc., que combatan los actos discriminatorios además de una transformación axiológica en el terreno del imaginario colectivo mediante un cambio cultural y educativo. 


\section{Aporofobia, el desprecio al Pobre, un desafío para la democracia}

Ahora se pasará a exponer el marco conceptual de Adela Cortina. Este es notablemente diferente al de Zepeda porque, aparte de que rastrea las causas de la discriminación no solamente a la cultura ni a los estigmas y prejuicios que ésta pueda contener, sino a bases biológicas (la manera en que nuestro cerebro funciona), Adela entiende y plantea el problema de la discriminación de forma distinta, incluso se ve en la necesidad de construir un vocablo nuevo para explicar esta nueva forma de entender el problema, el de aporofobia.

Respecto a este último punto, sobre la manera de entender y plantear el problema, Zepeda (2011) ve que las encuestas ENADIS 2005 y 2010 muestran con claridad que "nuestra sociedad abriga poderosos componente de misoginia, racismo, xenofobia y homofobia" (p.15). Caracteriza la cultura nacional por la heterofobia (un rechazo a lo diferente) y "por una sistemática negación de derechos a quienes llevan formas de vida no sujetas a los cánones de género, raciales, etarios, de capacidades, sexuales, morales o religiosos de la mayoría" (Zepeda, 2011, p.15). La propuesta de Cortina va encaminada a señalar matices en el rechazo a lo diferente, matices que muestran que la raíz de este rechazo no yace propiamente en la condición de ser diferente, si no en la condición de causar problemas desprendida de ser pobre. Para demostrar esto, hace una comparación de trato entre los extranjeros turistas y extranjeros inmigrantes y refugiados políticos de España. Los primeros son acogidos con entusiasmo y hospitalidad, en cambio, los segundos se les recibe con rechazo inmisericordioso: "se les cierra las puertas, se levantan alambrados y murallas, se impide el traspaso de las fronteras" (2017, p.5). Este rechazo y aversión no surge de su mera característica de ser extranjeros ni por que sean de otra raza o etnia, sino lo que molesta es que sean pobres, que vayan a complicar la vida, que no lleven al parecer recursos, sino problemas. Dado al hecho de que no hay palabra para referir este tipo de rechazo, aversión, temor y desprecio hacia el pobre, Cortina propone el término "aporofobia" que permite entender los actos discriminatorias desde un nuevo ángulo que consiste en comprender la discriminación en base a la condición de ser pobre, es decir, no en todos lo casos se rechaza o se expresa aversión por ser diferente o por el hecho de ser mujer, indígena, niña/o, discapacitado, joven, viejo, etc. La novedosa forma de entender el problema de la 
discriminación de Cortina consiste en ver una raíz común a las actitudes de rechazo de la xenofobia, misoginia, cristianofobia, islamofobia o la homofobia. Esta raíz común residen en la aporofobia: rechazo al pobre.

La exclusión que sufre el pobre no se limita a la de trato, ni a derechos, libertades y oportunidades socialmente relevante. Su exclusión sobrepasa estos ámbitos, "se le excluye de un mundo construido sobre el contrato político, económico o social, de ese mundo del dar y el recibir, en el que solo pueden entrar los que parecen tener algo interesante que devolver como retorno" (p.6). Esto lo explica Cortina más detalladamente:

Ante cualquier oferta explícita o implícita, la pregunta que se hace así mismo el destinatario es "¿y yo que gano con eso?”. Somos seres de carencia y necesitamos suplirla con educación, pero también con lo que los demás pueden darnos. De esta necesidad nace el Estado de Derecho, que dice asegurarnos protección si cumplimos con nuestros deberes y oportunidades. De ella nacen las grandes instituciones del mundo político, económico y cultural, con el compromiso de arropar a los ciudadanos, que siempre son vulnerables. Pero los pobres parecen quebrar este juego del toma y daca, porque nuestra mente calculadora percibe que no van a traer más que problemas a cambio y por eso prospera la tendencia a excluirlos (p.7).

Como se observa, la causa de la exclusión al pobre ya no se rastrea solamente a estigmas y prejuicios en el imaginario social/cosmovisión, su causa reside en la misma condición de ser pobre, las consecuencias que se desprenden de esto para los otros. No se señalan únicamente la cultura y sus prejuicios y estigmas como los motores sociales del rechazo; el motor de las actitudes de rechazo reside también en las condiciones del cerebro mismo de responder al medio. De aquí, se basa su tesis más escalofriante y de la cual, la causa de la aporofobia supera las condiciones culturales: "como actitud [la aforobofia] tiene alcance universal: todos los seres humanos son aporófobos, y esto tiene raíces cerebrales, pero también sociales [...]" (p.7). Tras entender el problema de la discriminación y sus causas de esta manera, su propuesta para modificar la condición aporófica del cerebro es 
mediante una educación que promueva la compasión. Para entender esta solución que ella propone es indispensable conocer el por qué nuestro cerebro es aporofobo.

Antes de desarrollar este punto, es importante hacer unas precisiones. Cortina anuda a la definición de pobreza como carencia de los medios necesarios para sobrevivir. La caracterización de Amartyan Sen define la pobreza como la falta de libertad, imposibilidad de llevar a cabo los planes de vida que una persona tenga razones para valorar. La aporofobia, tomada como actitud vital, "es desprecio y rechazo en cada caso de los peor situados, que pueden serlo económicamente, pero también socialmente" (p.27). Esta tendencia parece inscrita en la naturaleza humana, sin embargo, Cortina se da a la tarea de descubrir sus raíces profundas, tratar de investigar sus causas y descubrir caminos por los que cada persona y cada sociedad puede modificarlas.

\section{El cerebro y la aporofobia}

De las diferentes versiones de la naturaleza del cerebro humano, Cortina parte de la interpretación que lo concibe como un sistema autónomamente activo en interacción social constante que realiza valoraciones inevitablemente para posibilitar la supervivencia. Estas tendencias valorativas constituyen la identidad innata de los seres humanos. Estas plantean problemas a los largo de la vida porque a menudo entran en contradicción entre sí por ser a la vez sociales e individualistas.

Las tendencias que entran en conflicto son fundamentalmente el auto interés, la orientación de control, la disociación, la simpatía selectiva, la empatía y la xenofobia; así, la identidad neuronal nos hace sociales a la ves que individualistas.

Las bases cerebrales de la conducta xenófoba radican en la tendencia valorativa del autointerés y en la orientación de control. La primera refiere a un afán de sobrevivir que se expresa biológicamente en el deseo de estar bien alimentado, de sentirse seguro, de reproducirse etc. Este interés de supervivencia conlleva a controlar el entorno inmediato y a buscar lo familiar, la seguridad, a preferir lo conocido. Cierta seguridad es requerida en aras de desarrollarse de una forma sana. En efecto, el miedo a los extranjeros es algo completamente natural. 
La raíz de la aporofobia reside en la tendencia de la disociación que cuando una circunstancia nos perturba se cuenta con este mecanismo. Éste consiste en distanciarse de cosas que desagradan. Cortina señala que el auto interés lleva a rechazar la información que nos perturba, se trate de acontecimientos o de personas.

En base a esto, las emociones que conducen a prejuicios raciales y culturales tendrían como función, desde el punto de vista evolutivo, detectar las diferencias que podrían señalar un riesgo o un peligro, e incitar a retirarse o a agredir.

Siguiendo la lógica de la selección natural, la tendencia de la simpatía y empatía son adaptativas y los seres altruistas sobreviven en la medida en que van acorde a atender al interés propio -obsérvese que aunque son tendencias sociales, en la raíz yace el autointerés. El autointerés es la tendencia que permite desarrollar tendencias sociales. En efecto, una forma de superar el rechazo al extranjero es la expectativa de reciprocidad que entabla relaciones cooperativas en que ambas partes reciben algo a cambio. La cooperación es posible porque va acorde al interés de supervivencia. Cabe decir que una manera de superar la xenofobia es mediante la expectativa de reciprocidad ya sea directa o indirecta.

Y sucede que, al comprobar que el juego de dar y recibir resulta beneficioso para el grupo y para los individuos que lo componen, este juego ha ido cristalizando en normas de reciprocidad indirecta que forman el esqueleto sobre el que se sustenta la encarnadura de las sociedades contractualistas en las que vivimos, regida por el Principio de Intercambio (p.53).

Vimos como una tendencia evaluativa cerebral que es el rechazo al extranjero puede ser superada mediante el reforzamiento de otra tendencia: la expectativa de reciprocidad, porque ésta atiende al final de cuentas el autointerés, lo cual ha desembocado en sociedades contractualistas. Sin embargo, en este avance para superar la discriminación, aún queda sin resolver la tendencia valorativa de disociación que desemboca en la aporofobia: "desgraciadamente, [...] en la sociedad contractualista y cooperativa del intercambio se excluye [...] al que no entra en el juego del intercambio, porque no parece que pueda ofrecer ningún beneficio como retorno" (p.54). El principal desafío de la aporofobia 
es que tomar en cuenta al pobre implica perder capacidad adaptativa biológica social, siguiendo la lógica de la selección natural. Sin embargo, "el cerebro está dotado de una enorme plasticidad que nos permite modularlo a lo largo de la vida" (p.49). Cortina señala que la vía para reducir e incluso eliminar las fobias está en reforzar otras tendencias evaluativas universales como la tendencia de cuidar de otros. Específicamente en la aporofobia, las dos rutas para disminuirla, dice, es "hacia el reconocimiento recíproco de la dignidad y hacia la compasión, que rompe barreras y se extiende universalmente" (p.55).

\section{Conclusiones}

Las tendencias evaluativas universales se encuentran en la raíz de las fuerzas que nos llevan a actuar siempre atendiendo el interés de la supervivencia.

Al parecer, las emociones que conducen a prejuicios raciales y culturales tienen su base en parte en emociones sociales que, desde el punto de vista evolutivo, servían para detectar las diferencias que podían señalar un riesgo o un peligro, e incitar a retirarse o a agredir (Cortina, 2017, p.49).

En este sentido, cabe decir que los estigmas y prejuicios son un producto de las tendencias evaluativas universales. La propuesta de Cortina nos parece muy prometedora, porque ella propone atender ese terreno de donde se producen las actitudes de desprecio, exclusión, de rechazo, etc. Las observaciones de Cortina nos llevan a entender que la tarea de la antidiscriminación implica ir en contra de la biología del cerebro mismo. Nos propone negociar con las tendencias evaluativas del cerebro de tal modo que el sueño de la igualdad de trato sea posible desde la configuración de las tendencias en el cerebro (Cortina, 2017, pp.50,54 y 55).

La propuesta de Zepeda también parece prometedora porque, desde su marco conceptual, la desigualdad de trato deriva de estigmas y prejuicios que albergan en la cosmovision/imaginario social, desigualdad de trato que produce prácticas sistematizadas tanto informales como formales; en base a este entendimiento, resulta prometedor que una transformación del imaginario social 
mediante la educación, y la creación de instituciones promotoras de la igualdad de trato que cambien las prácticas sistematizadas de discriminación sean las vías que un proyecto antidiscriminatorio deba emprender. Sin embargo, el marco conceptual de Zepeda no nos explica el porqué en la cultura se formaron tales estigmas y prejuicios. Su marco conceptual nos diría que los estigmas y prejuicios provienen de la cultura, ¿pero qué creo esa cultura discriminatoria? En este punto es que el marco conceptual de Cortina abona al de Zepeda para comprender ese hueco, a la vez que proporciona directrices del tipo de educación en específico (de compasión y cuidado del otro) que ha de difundirse de modo que las fuerzas cerebrales que producen los estigmas y prejuicios sean modificados acorde al fin de la igualdad de trato. También, se encuentra riesgoso en el marco conceptual de Zepeda, que se deje fuera de la comprensión del fenómeno de la discriminación el ámbito de las tendencias evaluativas del cerebro porque sería desatender la raíz productora de las prácticas discriminatorias. Suena plausible que el insertar prácticas de igualdad promuevan más prácticas de este tipo y también que la erradicación de prejuicios y estigmas del imaginario social detenga la producción de actos discriminatorios, pero si la cultura se formó discriminatoria, es lógico que algo la produjo así, pues como dice la frase de Parménides "nada surge de la nada" ¿Qué asegura que ese algo -desconocido en el marco conceptual de Zepeda- no vuelva a producir la cultura discriminatoria? La propuesta de Cortina que señala y define eso que crea las emociones sociales que llevan a excluir, rechazar, despreciar y propone medidas para transformar ese algo que son las tendencias evaluativas cerebrales (Cortina, 2017, pp. 50, 54 y 55). Por esto creemos que la propuesta de Cortina es significativa para proyectos que pretendan eliminar la discriminación.

\section{Referencias:}

Arenas, V. (2017). Guadalajara Firma Convenio con Conapred. El Informador. recuperado en: https://www.informador.mx/jalisco/Guadalajara-firma-convenio-con-Conapred-201710260116.html

Baylón, L. (08 de Agosto del 2018). México no cree (casi nada) en los jóvenes y con piel morena las cosas no mejoran: ENADIS [huffingtonpost]. Recuperado de: 
https://www.huffingtonpost.com.mx/2018/08/07/mexico-no-cree-nada-o-casi-nada-en-losjovenes-y-con-una-piel-morena-las-cosas-no-mejoran-encuesta-nacional-sobrediscriminacion a 23497887/

Congreso del Estado de Jalisco. (11 de octubre del 2016). Ley Estatal para Promover la Igualdad,

Prevenir y Eliminar la Discriminación en el Estado de Jalisco. [25873/LXI/16] DO:

https://www.conapred.org.mx/leyes/Ley Estatal para Promover la Igualdad Prevenir y Eli minar la Discriminacion en JaliscoAx.pdf

Consejo Nacional para Prevenir la Discriminación. (2011). Encuesta Nacional sobre discriminación:

Enadis 2010, resultados generales. Recuperado en:

https://www.conapred.org.mx/documentos_cedoc/Enadis-2010-RG-Accss-002.pdf

Consejo para Prevenir y Eliminar la Discriminación de la Ciudad de México. (2013). (s.f.). Antología de política pública antidiscriminatoria 2013-2017. Recuperado de:

https://copred.cdmx.gob.mx/storage/app/uploads/public/5ab/409/6d7/5ab4096d7ca2d68553

\section{8.pdf}

Consejo para Prevenir y Eliminar la Discriminación de la Ciudad de México. (s/f). Derecho a la igualdad y no discriminación. Recuperado de

https://copred.cdmx.gob.mx/storage/app/uploads/public/5a3/807/7c9/5a38077c94e4541319 5450.pdf

Cortina, A. (2017). Aporofobia, el rechazo al pobre: Un desafío para la democracia. Barcelona, España: Paidós.

El Informador (2018). Jalisco se mantiene en tercer lugar nacional en quejas por discriminación. El informador. Recuperado en: https://www.informador.mx/jalisco/Jalisco-se-mantiene-en-eltercer-lugar-nacional-en-quejas-por-discriminacion-20180114-0026.html

Gutiérrez, E. M. (2018). Persiste la deuda con los pueblos indígenas. El Milenio. Recuperado en: http://www.milenio.com/politica/comunidad/persiste-la-deuda-con-los-pueblos-indigenas Instituto Nacional de Estadísticas y Geografía. (2018a). Encuesta Nacional sobre Discriminación 2017, ENADIS, Diseño conceptual. Recuperado de: 
http://www.beta.inegi.org.mx/contenidos/proyectos/enchogares/especiales/enadis/2017/doc Lenadis2017 diseno conceptual.pdf

Instituto Nacional de Estadísticas y Geografía. (2018b). Nota técnica: Encuesta nacional sobre discriminación (Enadis 2017). COMUNICADO DE PRENSA NÚM. 346/18. Recuperado en:

http://www.beta.inegi.org.mx/contenidos/saladeprensa/boletines/2018/estsociodemo/enadis 2017 08.pdf

Marco Teórico. (s.f.). Recuperado de: https://www.marcoteorico.com/curso/50/definicion-de-marcoteorico.

Merino, M. y Vilalta, C. (2014). En busca de indicadores sobre la desigualdad de trato en el gasto público federal (Una aproximación de política pública al estudio sobre discriminación en México). Hacia una razón antidiscriminatoria. Estudios analíticos y normativos sobre la desigualdad de trato (438). México: Conapred.

Morente, M. (2013). Lecciones preliminares de filosofía. México: Época.

Naciones Unidas. (2015). Declaración universal de derechos humanos. Recuperado de:

http://www.un.org/es/documents/udhr/UDHR booklet SP web.pdf

Congreso de la Unión. (10 de junio de 2011). Decreto por el que se modifica la denominación del Capítulo I del Título Primero y reforma diversos artículos de la Constitución Política de los Estados Unidos Mexicanos. [s. No. de decreto]. DO:

http://dof.gob.mx/nota detalle.php?codigo=5194486\&fecha=10/06/2011

Congreso de la Unión. (14 de agosto del 2001). DECRETO por el que se aprueba el diverso por el que se adicionan un segundo y tercer párrafos al artículo 10., se reforma el artículo 2o., se deroga el párrafo primero del artículo 4o.; y se adicionan un sexto párrafo al artículo 18, y un último párrafo a la fracción tercera del artículo 115 de la Constitución Política de los Estados Unidos Mexicanos. [s. No. de decreto]. DO:

http://www.dof.gob.mx/nota_detalle.php?codigo=762221\&fecha=14/08/2001

Zepeda, J. (2011). La otra desigualdad: La discriminación en México. D.F, México: Conapred. 